\title{
LncRNA MIR4435-2HG is downregulated in osteoarthritis and regulates chondrocyte cell proliferation and apoptosis
}

\author{
Yu Xiao ${ }^{1 *+} \mathbb{D}$, Yucheng $\mathrm{BaO}^{2+}$, Liang Tang ${ }^{2}$ and Li Wang ${ }^{2}$
}

\begin{abstract}
Purpose: MIR4435-2HG is an oncogenic IncRNA in gastric cancer and lung cancer. Our preliminary microarray data showed that MIR4435-2HG was downregulated in osteoarthritis plasma specimen, indicating the possible involvement of MIR4435-2HG in osteoarthritis.
\end{abstract}

Results: MIR4435-2HG was downregulated in plasma of osteoarthritis than in plasma of healthy controls. Reduced levels of MIR4435-2HG expression effectively distinguished osteoarthritis patients from the control group. Expression levels of MIR4435-2HG increased after treatment. Overexpression of MIR4435-2HG promoted, while MIR4435-2HG knockdown inhibited the proliferation of chondrocytes. In contrast, MIR4435-2HG overexpression inhibited, while MIR4435-2HG knockdown promoted the apoptosis of chondrocytes.

Conclusion: MIR4435-2HG is downregulated in osteoarthritis and regulates chondrocyte cell proliferation and apoptosis.

Keywords: Osteoarthritis, IncRNA MIR4435-2HG, Proliferation, Apoptosis

\section{Introduction}

Osteoarthritis (OA) is a common clinical degenerative joint disease that is characterized by subchondral bone thickening, degradation of articular cartilage, and the formation of osteophytes [1]. Osteoarthritis now has become a major problem of public health worldwide, and more than half of people aged older than 65 years are suffering from this disease [2]. OA is associated and is closely correlated with homeostatic imbalance, which results from aging [3]. With the increase in people's life expectancy, the incidence of osteoarthritis is predicted to be further increased in the near future [4]. Although several risk factors have been characterized for osteoarthritis, the pathogenesis of this disease is still unclear and definitive cure is also not available [5].

Although long non-coding RNAs (lncRNAs) have no protein-coding capacity, there are key players in the development of human diseases [6,7]. We carried out a

\footnotetext{
* Correspondence: omammeaz715196438@126.com

† Yu Xiao and Yucheng Bao contributed equally to this work.

${ }^{1}$ Department of Orthopaedic Surgery, Tianjin Hospital, No.406 Jiefang South

Road, Hexi, Tianjin City 300211, People's Republic of China

Full list of author information is available at the end of the article
}

genome-wide transcriptome analysis of differentially expressed gene in plasma of osteoarthritis patients. Our preliminary data showed that MIR4435-2HG was downregulated in osteoarthritis (data not shown). MIR4435-2HG has been proved to be an oncogenic lncRNA in gastric cancer and lung cancer $[8,9]$. We showed that MIR4435-2HG was downregulated in osteoarthritis and regulated chondrocyte cell proliferation and apoptosis.

\section{Materials and methods}

Research subjects

A total of 78 osteoarthritis (44 cases of knee joint and 34 cases of hip joint; 40 males and 38 females; 60 to 78 years old; mean age $69.0 \pm 6.0$ years) patients and 58 healthy volunteers (30 males and 28 females; 60 to 77 years; mean age $68.8 \pm 5.9$ years) were enrolled in Tianjin Hospital from January 2015 to January 2017 to serve as research subjects. All patients were diagnosed by joint fluid analysis, which revealed the existence of inflammation and X-rays, which showed the narrowing of the space between the bones, an indicator of cartilage loss. Patients' inclusion criteria: (1) new cases of osteoarthritis 
(stages 3 and 4) (2) patients provided informed consent. Patients' exclusion criteria: (1) patients complicated with other diseases, such as chronic inflammatory diseases other than osteoarthritis; (2) patients failed to complete treatment; (3) patients who had been treated before admission or currently under medication. The 58 healthy volunteers received systemic physical examinations at the physical health center of Tianjin Hospital, and all parameters were within the normal range. The research has been carried out in accordance with the World Medical Association Declaration of Helsinki, and this study was approved by the Ethics Committee of Tianjin Hospital.

\section{Plasma specimens, synovial fluid treatment, and cell line} Blood $(5 \mathrm{ml})$ was extracted from both patients and healthy controls 1 day after admission under fasting condition. Synovial fluid was collected from all patients and controls (33 cases of hip and 25 cases of the knee). All patients received treatments including exercise, reducing joint burden, and non-steroidal anti-inflammatory drugs (NSAIDs) such as naproxen. Inflammation was inhibited after 1-month treatment, which was revealed by joint fluid analysis. Blood $(5 \mathrm{ml})$ was also extracted at 1 and 3 months after treatment from each patient under fasting condition. Blood samples were used to prepare plasma samples.

Thirteen cases of synovial membrane from osteoarthritis-affected joints and 8 cases of synovial membrane from non-osteoarthritis-affected joints were obtained from the specimen library of Tianjin Hospital.

Primary chondrocytes (402-05A, Sigma-Aldrich) were used in this study to perform in vitro experiments. Cell culture was performed according to the instructions from Sigma-Aldrich.

\section{QPCR}

To detect the expression of lncRNA MIR4435-2HG, total RNAs were extracted using Trizol reagent (Invitrogen, USA). After reverse transcription (SuperScript IV Reverse Transcriptase kit, Thermo Fisher Scientific., lnc.), SYBR ${ }^{\bullet}$ Green Quantitative RT-qPCR Kit (Sigma-Aldrich) was used to prepare qPCR mixtures. Primers of MIR44352HG and endogenous control GAPDH were from Sangon (Shanghai, China). Sequences of primers were: $5^{\prime}$-TGAT AAAGGGCTCTGAAAGC-3' (Forward) and 5'-CACG ATGCCTTCACCAGTGT-3' (reverse) for MIR44352HG; 5'-CTGACTTCAACAGCGACAC-3' (forward) and $5^{\prime}$-TAGCCAAATTCGTTGTCATAC-3' (reverse) for GAPDH. According to $2^{-\triangle \Delta C T}$ method, expression of MIR4435-2HG was normalized to GAPDH.

\section{Cell transfection}

MIR4435-2HG-expression vector and empty vectors (pEGFP-C3) were from Sangon (Shanghai, China).
MIR4435-2HG siRNA and negative control (NC) siRNA were from GenePharma (Shanghai, China). Lipofectamine 2000 reagent (Thermo Fisher Scientific., lnc.) was used for all cell transient transfections with $10 \mathrm{nM}$ vector (empty vector as $\mathrm{NC}$ group) or $35 \mathrm{nM}$ siRNAs (NC siRNA as NC group). Cells without transfection were control cells. Cells were collected for subsequent experiments at $24 \mathrm{~h}$ after transfection.

\section{Cell proliferation assay}

Cells were collected at $24 \mathrm{~h}$ after transfection, and $4 \times 10^{4}$ cells were mixed with $1 \mathrm{ml}$ cell culture medium to make single cell suspensions. Cells were cultivated in a 96-well plate $\left(100 \mu \mathrm{l}\right.$ each well) at $37^{\circ} \mathrm{C}$, followed by the addition of $10 \mu \mathrm{l} \mathrm{CCK-} 8$ solution (Sigma-Aldrich) at $3 \mathrm{~h}$ before the end of cell culture. After that, $10 \mu \mathrm{l}$ DMSO was added, and the measurement of OD values was performed at $450 \mathrm{~nm}$.

\section{Cell apoptosis assay}

Cells were collected at $24 \mathrm{~h}$ after transfection and $4 \times 10^{4}$ cells were mixed with $1 \mathrm{ml}$ serum-free cell culture medium to make single cell suspensions. A 6-well plate was used to cultivate cells ( $2 \mathrm{ml}$ per well) for $48 \mathrm{~h}$. After that, cells were digested by $0.25 \%$ trypsin and stained by Annexin V-FITC (Dojindo, Japan) as well as propidium iodide (PI). Apoptotic cells were separated by flow cytometry.

\section{Statistical analysis}

All experiments were performed in triplicate manner and data were recorded as mean \pm standard deviation. Comparisons of expression levels of MIR4435-2HG between two groups were done by unpaired $t$ test. Comparisons of expression levels of MIR4435-2HG in patients between different time points were performed by paired $t$ test. Comparisons of cell apoptosis and proliferation data among the three groups were done by ANOVA (one-way) and Tukey test. The diagnostic analysis was performed through ROC curve analysis with healthy controls as true negative cases and osteoarthritis patients as true positive cases. DP $<0.05$ was statistically significant.

\section{Results}

Plasma MIR4435-2HG was downregulated in osteoarthritis patients

RT-qPCR was performed to measure levels of MIR4435$2 \mathrm{HG}$ in plasma of osteoarthritis patients and healthy controls. As shown in Fig. 1a, plasma levels of MIR44352HG were significantly lower in osteoarthritis patients than in the control group $(p<0.05)$. Moreover, MIR4435-2HG expression in 13 cases of synovial membrane from osteoarthritis-affected joints was also downregulated 
A

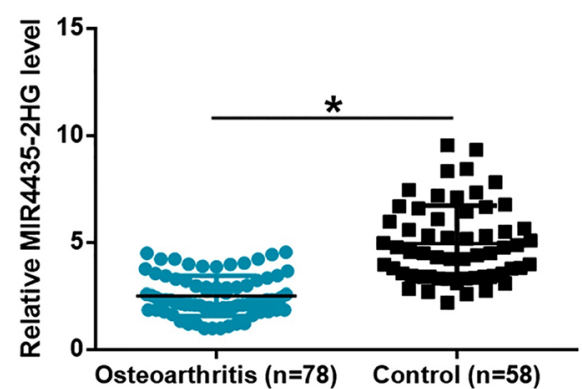

B

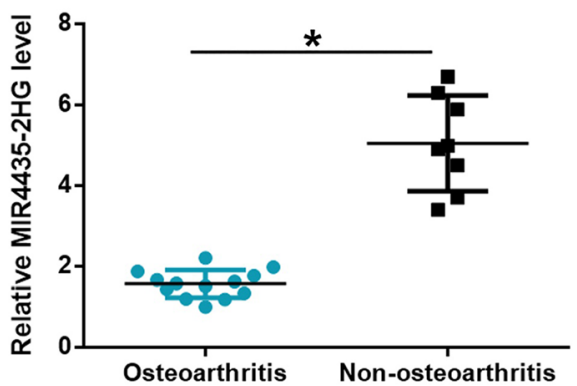

Fig. 1 Plasma MIR4435-2HG was downregulated in osteoarthritis patients. RT-qPCR results showed that plasma levels of MIR4435-2HG in osteoarthritis patients and in healthy controls (a). In addition, MIR4435-2HG expression in 13 cases of synovial membrane from osteoarthritisaffected joints was also downregulated comparing to 8 cases of synovial membrane from non-osteoarthritis-affected joints (b). ( $\left.{ }^{*} p<0.05\right)$

comparing to 8 cases of synovial membrane from nonosteoarthritis-affected joints (Fig. 1b). These data suggest that downregulation of MIR4435-2HG is likely involved in osteoarthritis.

\section{Downregulation of MIR4435-2HG in join fluid effectively} distinguished osteoarthritis patients from the control group MIR4435-2HG in join fluid was also measured by qPCR. It was observed that expression levels of MIR4435-2HG in join fluid were also significantly lower in osteoarthritis patients than in the control group (Fig. $2 \mathrm{a}, p<0.05$ ). The diagnostic analysis was performed through ROC curve analysis. As shown in Fig. 2, area under the curve was 0.96 , with a standard error of 0.016 and $95 \%$ confidence interval of $0.92-0.99$ (Fig. $2 \mathrm{~b}, p<0001$ ). It is suggested that downregulation of MIR4435-2HG may serve as a potential diagnostic biomarker for osteoarthritis.

Expression levels of MIR4435-2HG increased after treatment Plasma levels of MIR4435-2HG were measured before treatment as well as 1 and 3 months after the beginning of treatment. Compared with pretreatment levels, plasma levels of MIR4435-2HG were significantly increased at 1 and 3 months after the beginning of treatment (Fig. 3, $p<0.05$ ). Comparing to plasma levels of MIR4435-2HG at 1 after the beginning of treatment, plasma levels of MIR4435-2HG were significantly increased at 3 months after the beginning of treatment (Fig. 3, $p<0.05$ ).

\section{MIR4435-2HG regulates the proliferation and apoptosis of chondrocytes}

Overexpression and knockdown of MIR4435-2HG were reached after transfection in chondrocytes (Fig. 4a, $p<0.05)$. Overexpression of MIR4435-2HG promoted, while MIR4435-2HG knockdown inhibited the proliferation of cells of chondrocytes (Fig. $4 \mathrm{~b}, p<0.05$ ). In contrast, MIR4435-2HG overexpression inhibited, while MIR4435-2HG knockdown promoted the apoptosis of chondrocytes (Fig. 4c, $p<0.05$ ). Therefore, MIR4435-2HG regulated chondrocyte cell proliferation and apoptosis.
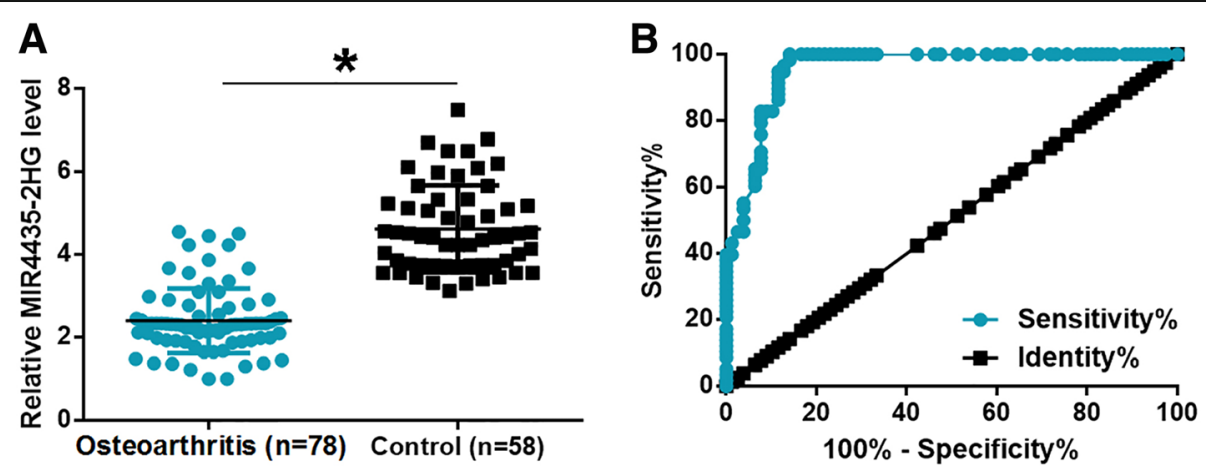

Fig. 2 Downregulation of MIR4435-2HG effectively distinguished osteoarthritis patients from healthy controls. MIR4435-2HG in joint fluid was also measured by qPCR. It was observed that expression levels of MIR4435-2HG in join fluid were also significantly lower in osteoarthritis patients than in the control group (a). ROC curve analysis showed that downregulation of MIR4435-2HG effectively distinguished osteoarthritis patients from healthy controls $(\mathbf{b}) .\left(^{*} p<0.05\right)$ 


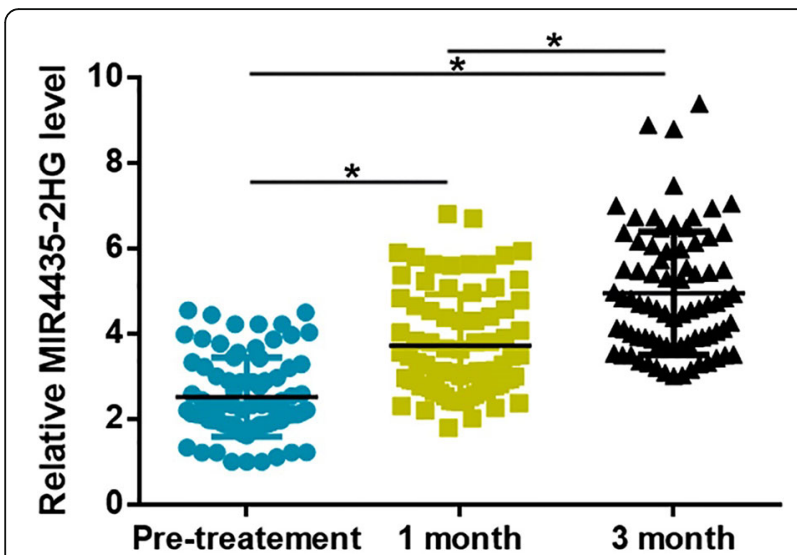

Fig. 3 Expression levels of MIR4435-2HG increased after treatment. RT-qPCR results showed that expression levels of MIR4435-2HG increased significantly after treatment $\left({ }^{*} p<0.05\right)$

\section{Discussion}

Osteoarthritis is a common clinical degenerative joint disease with unknown causes. We found that MIR44352HG was downregulated in osteoarthritis and the overexpression of MIR4435-2HG may regulate the behaviors of chondrocytes.

Although the pathogenesis of osteoarthritis is still unclear, the development and progression of this disease is always accompanied by changes in expression pattern of a large set of genes $[10,11]$, such as lncRNAs Several differentially expressed lncRNAs, such as lncRNA lncRNA HOTAIR and IncRNA UFC1 have been proved to be essential players in osteoarthritis $[12,13]$. LncRNA HOTAIR promotes the apoptosis of chondrocytes, which in turn aggregates disease condition [12]. In contrast, lncRNA UFC1 was downregulated in osteoarthritis, and the upregulation of IncRNA UFC1 promotes the

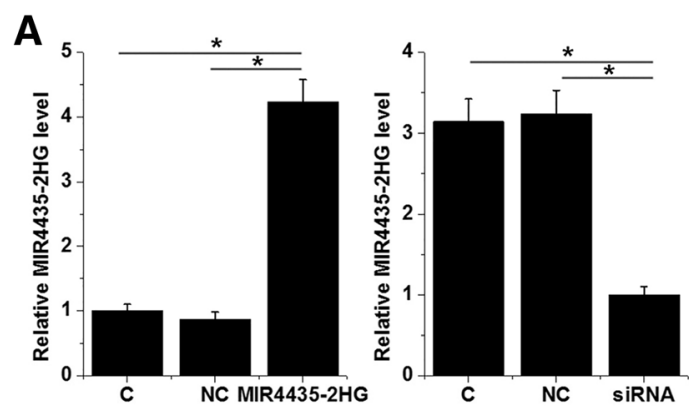

B
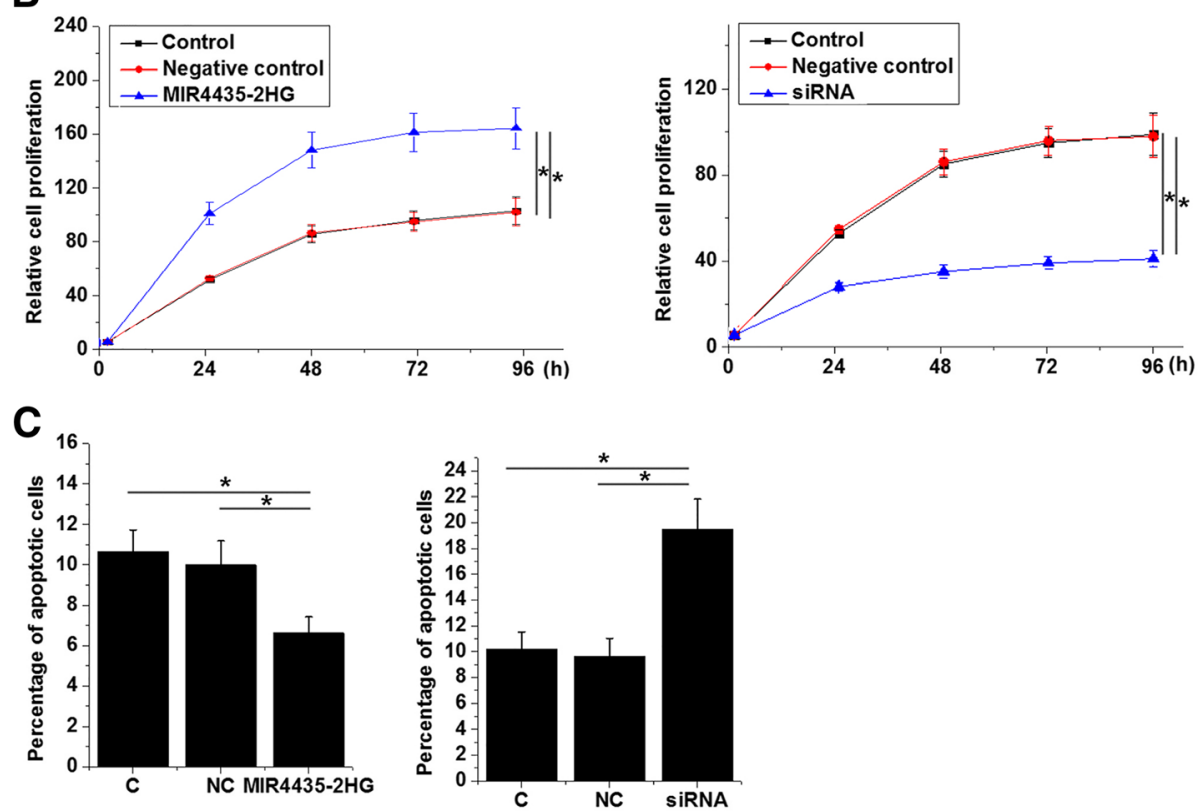

Fig. 4 MIR4435-2HG regulates the proliferation and apoptosis of chondrocytes. Overexpression and knockdown of MIR4435-2HG were reached after transfection into chondrocytes (a). Overexpression of MIR4435-2HG promoted, while MIR4435-2HG knockdown inhibited the proliferation of chondrocytes (b). In contrast, MIR4435-2HG overexpression inhibited, while MIR4435-2HG knockdown promoted the apoptosis of chondrocytes (c) $\left.{ }^{*} p<0.05\right)$ 
proliferation of chondrocyte [13]. Our study first reported the downregulation of plasma circulating MIR4435-2HG could be used to distinguish osteoarthritis patients from the control group. Therefore, plasma MIR4435-2HG may serve as a potential therapeutic biomarker for osteoarthritis. However, more clinical studies are needed to further investigate the diagnostic sensitivity and specificity.

Cartilage cellularity, which is critical for joint function, is reduced in osteoarthritis [14]. It is known that the reduced cartilage cellularity in osteoarthritis patients is closely correlated with chondrocyte death $[15,16]$. Chondrocyte apoptosis inhibition is a potential approach for the treatment of osteoarthritis [17]. In the present study, we showed that MIR4435-2HG promoted the proliferation but inhibited the apoptosis of chondrocytes. Therefore, overexpression of MIR4435-2HG may serve as a promising therapeutic target for the treatment of osteoarthritis. However, more clinical trials and in vivo animal model studies are needed to further test our hypothesis.

\section{Conclusion}

In conclusion, MIR4435-2HG is downregulated in osteoarthritis and may serve as a therapeutic target for osteoarthritis by promoting chondrocyte cell proliferation and inhibiting cell apoptosis.

\section{Abbreviations \\ IncRNAs: Long non-coding RNAs; NSAIDs: Non-steroidal anti-inflammatory drugs; OA: Osteoarthritis}

\section{Acknowledgements}

Not applicable.

\section{Authors' contributions}

YX and YB designed and carried out the study. LT and LW participated in the experiments and statistical analysis. YX and YB wrote the manuscript. YX revised the manuscript. All authors read and approved the final manuscript.

\section{Funding}

This research received no specific grant from any funding agency in the public, commercial, or not-for-profit sectors.

\section{Availability of data and materials}

The analyzed datasets generated during the study are available from the corresponding author on reasonable request.

\section{Ethics approval and consent to participate}

The present study was approved by the Ethics Committee of Tianjin Hospital. The research has been carried out in accordance with the World Medical Association Declaration of Helsinki. All patients and healthy volunteers provided written informed consent prior to their inclusion within the study.

\section{Consent for publication}

Not applicable.

\section{Competing interests}

The authors declare that they have no competing interests.

\section{Author details}

${ }^{1}$ Department of Orthopaedic Surgery, Tianjin Hospital, No.406 Jiefang South Road, Hexi, Tianjin City 300211, People's Republic of China. ${ }^{2}$ Department of
Orthopaedic Surgery, Tianjin Haihe Hospital, 890 Jingu Road, Shuanggang Zhen, Jinnan, Tianjin 300350, People's Republic of China.

Received: 22 March 2019 Accepted: 11 July 2019

Published online: 06 August 2019

References

1. Hayami T, Pickarski M, Zhuo Y, Wesolowski GA, Rodan GA, Duong LT. Characterization of articular cartilage and subchondral bone changes in the rat anterior cruciate ligament transection and meniscectomized models of osteoarthritis. Bone. 2006:38:234-43.

2. Bijlsma JWJ, Berenbaum F, Lafeber FPJG. Osteoarthritis: an update with relevance for clinical practice. Lancet. 2011;377(9783):2115-26.

3. Gossan N, Boot-Handford R, Meng QJ. Ageing and osteoarthritis: a circadian rhythm connection. Biogerontology. 2015;16(2):209-19.

4. Rahman MM, Cibere J, Goldsmith $\mathrm{CH}$, et al. Osteoarthritis incidence and trends in administrative health records from British Columbia, Canada. J Rheumatol. 2014;41(6):1147-54.

5. Silverwood V, Blagojevic-Bucknall M, Jinks C, et al. Current evidence on risk factors for knee osteoarthritis in older adults: a systematic review and metaanalysis. Osteoarthr Cartil. 2015;23(4):507-15.

6. Fatica A, Bozzoni I. Long non-coding RNAs: new players in cell differentiation and development. Nat Rev Genet. 2014;15(1):7-21.

7. Shi $X$, Sun $M$, Liu $H$, et al. Long non-coding RNAs: a new frontier in the study of human diseases. Cancer Lett. 2013;339(2):159-66.

8. Ke D, Li H, Zhang Y, et al. The combination of circulating long noncoding RNAs AK001058, INHBA-AS1, MIR4435-2HG, and CEBPA-AS1 fragments in plasma serve as diagnostic markers for gastric cancer. Oncotarget. 2017; 8(13):21516-25.

9. Qian $\mathrm{H}$, Chen L, Huang J, et al. The IncRNA MIR4435-2HG promotes lung cancer progression by activating beta-catenin signalling. J Mol Med (Berl). 2018;96(8):753-64.

10. Fu M, Huang G, Zhang Z, et al. Expression profile of long noncoding RNAs in cartilage from knee osteoarthritis patients. Osteoarthr Cartil. 2015;23(3): 423-32.

11. Xing D, Liang J, Li Y, et al. Identification of long noncoding RNA associated with osteoarthritis in humans. Orthop Surg. 2014;6(4):288-93.

12. Zhang $C$, Wang $P$, Jiang $P$, et al. Upregulation of IncRNA HOTAIR contributes to IL-1ß-induced MMP overexpression and chondrocytes apoptosis in temporomandibular joint osteoarthritis. Gene. 2016;586(2):248-53.

13. Zhang G, Wu Y, Xu D, et al. Long noncoding RNA UFC1 promotes proliferation of chondrocyte in osteoarthritis by acting as a sponge for miR34a. DNA Cell Biol. 2016;35(11):691-5.

14. Huch K. Knee and ankle: human joints with different susceptibility to osteoarthritis reveal different cartilage cellularity and matrix synthesis in vitro[J]. Arch Orthop Trauma Surg. 2001;121(6):301-6.

15. Hwang $\mathrm{H}, \mathrm{Kim} \mathrm{H}$. Chondrocyte apoptosis in the pathogenesis of osteoarthritis. Int J Mol Sci. 2015;16(11):26035-54.

16. Thomas CM, Fuller CJ, Whittles CE, et al. Chondrocyte death by apoptosis is associated with cartilage matrix degradation. Osteoarthritis Cartilage. 2007; 15(1):27-34

17. Delco ML, Bonnevie ED, Szeto HH, et al. Mitoprotection as a strategy to prevent chondrocyte death and cartilage degeneration following mechanical injury. Osteoarthr Cartil. 2016;24:S500-1.

\section{Publisher's Note}

Springer Nature remains neutral with regard to jurisdictional claims in published maps and institutional affiliations. 